\title{
Crystal structure of bis(diethylenetriamino)nickel(II) dinitrate, $\left[\mathrm{Ni}(\text { dien })_{2}\right]\left(\mathrm{NO}_{3}\right)_{2}$
}

\author{
H.-L. Zhu*,I, S.-Y. Li ${ }^{I}$, Y.-J. Pan ${ }^{\mathrm{I}}$ and K.-B. Yu ${ }^{\mathrm{II}}$ \\ 1 Wuhan Institute of Science and Technology, Department of Environmental and Chemical Engineering, Wuhan, 430073, P. R. China \\ II Chinese Academy of Science, Chengdu Center of Analysis and Measurement, Chengdu 610041, P. R.China
}

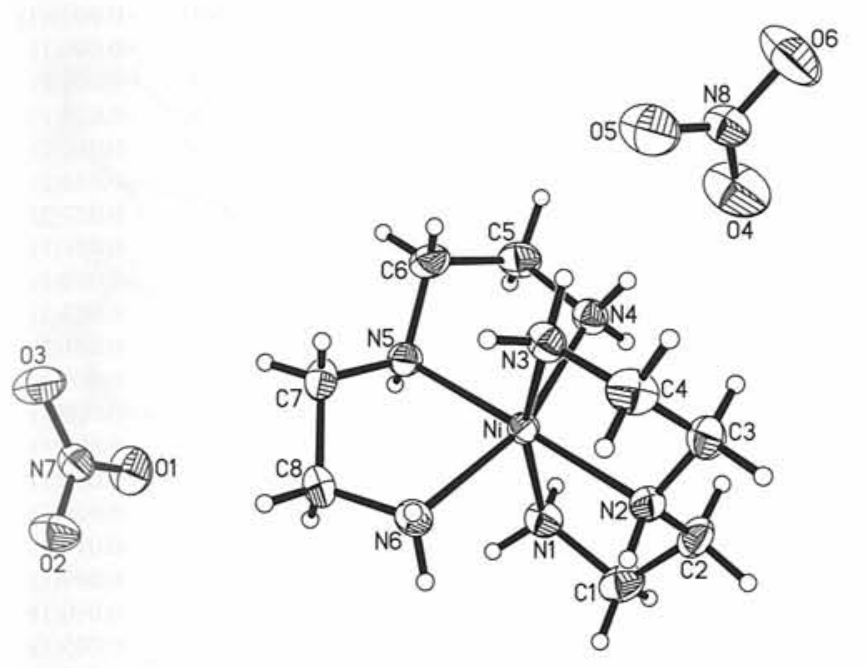

Abstract

$\mathrm{C}_{8} \mathrm{H}_{26} \mathrm{~N}_{8} \mathrm{NiO}_{6}$, monoclinic, $P 12_{1} / c 1$ (No. 14), $a=13.351(2) \AA$, $b=8.931(1) \AA, c=14.207(2) \AA, \beta=92.61(1)^{\circ}, V=1692.3 \AA^{3}$, $\mathrm{Z}=4, R_{\mathrm{gt}}(F)=0.030, w R_{\text {ref }}\left(F^{2}\right)=0.072, T=296 \mathrm{~K}$.

Source of material

An alcohol solution ( $5 \mathrm{ml})$ of dien $(2 \mathrm{mmol}, 206 \mathrm{mg}$ ) was added to an aqua-alcohol solution $(1: 1 \mathrm{v} / \mathrm{v}, 5 \mathrm{ml})$ of $\mathrm{Ni}\left(\mathrm{NO}_{3}\right)_{2} \cdot 6 \mathrm{H}_{2} \mathrm{O}$ (1 mmol, $291 \mathrm{mg})$. The resulting purple solution was stood still in air to evaporate about half of the solvents. Large blue prism crystals of $\mathrm{C}_{8} \mathrm{H}_{26} \mathrm{NiN}_{8} \mathrm{O}_{6}$ were collected by filtration, washed with alcohol and dried with $\mathrm{CaCl}_{2}$ in vacuum (yield $35 \%$ ). Analysis: found: C, $24.28 \%$; H, $6.90 \%$; N, $28.35 \%$; calc. for $\mathrm{C}_{8} \mathrm{H}_{26} \mathrm{NiN}_{8} \mathrm{O}_{6}: \mathrm{C}, 24.70 \%$; $\mathrm{H}, 6.74 \%$; N, 28.80\%).

\section{Discussion}

Since nickel is present in the active sites of several important classes of metalloproteins, and its potent application in the design and construction of new magnetic materials, study on nickel complexes is of great interest in various aspects of chemistry [1-4]. Molecular magnetism is one of the most active fields in modern inorganic chemistry, and materials exhibiting different magnetic properties have been the focus of many contemporary experimental and theoretical studies. A mononuclear nickel(II) complex with diethylenetriamine is reported here.

The crystal structure of the title complex shows that it comprises of a discrete mononuclear $\left[\mathrm{Ni}(\text { dien })_{2}\right]^{2+}$ cation and two nitrate anions. In each cation, the nickel(II) atom is coordinated by six nitrogen atoms from two dien molecules. The average $\mathrm{Ni}-\mathrm{N}$ bond distances

\footnotetext{
* Correspondence author (e-mail: hlzhu@ wist.edu.cn)
}

(2.148 $\AA$ for nickel(II) and primary nitrogens, and $2.086 \AA$ for nickel(II) and secondary nitrogen atoms) are comparable to those in the complexes we reported previously $[3,4]$. The nickel(II) atom has a greatly distorted octahedral environment with $\mathrm{N} 1, \mathrm{~N} 3$, $\mathrm{N} 4$ and N6 (the four primary nitrogen atoms) constituting the equatorial plane and the two secondary nitrogen atoms (N2 and N5) occupying the two axial positions. One ( $\angle \mathrm{N} 5-\mathrm{Ni}-\mathrm{N} 2=$ $178.72(8)^{\circ}$ ) of the three diagonal angles is close to $180^{\circ}$, and the other two diagonals $\left(\angle \mathrm{N} 6-\mathrm{Ni}-\mathrm{N} 4=162.44(8)^{\circ}\right.$ and $\left.\angle \mathrm{N} 1-\mathrm{Ni}-\mathrm{N} 3=162.54(8)^{\circ}\right)$ deviate from $180^{\circ}$, indicating that the coordination around the nickel(II) atom is a largely distorted octahedron. In order to minimize the steric effects, two dien molecules in the $\left[\mathrm{Ni}(\text { dien })_{2}\right]^{2+}$ cation are nearly perpendicular to each other. All the nitrogen atoms in dien molecules and four of the six oxygen atoms in the two nitrate anions participate in the formation of H-bonds (from 2.953(3) $\AA$ to 3.187(3) $\AA$ ), which join the complex into a three-dimensional structure.

Table 1. Data collection and handling.

Crystal:

Wavelength:

$\mu$ :

Diffractometer, scan mode:

$2 \theta_{\text {max }}$ :

$N(h k l)_{\text {measured, }} N(h k l)_{\text {unique: }}$

Criterion for $I_{\mathrm{obs}}, N(h k l)_{\mathrm{gt}}$ :

$N(\text { param })_{\text {refined: }}$

Program:

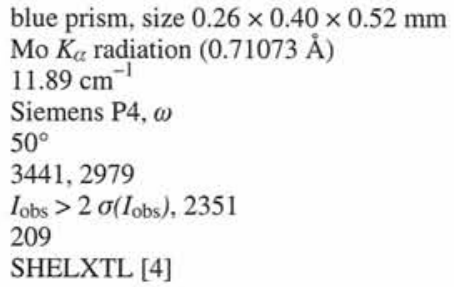

Table 2. Atomic coordinates and displacement parameters (in $\AA^{2}$ ).

\begin{tabular}{llllll}
\hline Atom & Site & $x$ & \multicolumn{1}{l}{$y$} & $U_{\text {iso }}$ \\
\hline $\mathrm{H}(1 \mathrm{~A})$ & $4 e$ & 0.7485 & 0.1484 & 0.2497 & 0.051 \\
$\mathrm{H}(1 \mathrm{~B})$ & $4 e$ & 0.8526 & 0.1230 & 0.2816 & 0.051 \\
$\mathrm{H}(2 \mathrm{~N})$ & $4 e$ & 0.6700 & -0.1273 & 0.4067 & 0.045 \\
$\mathrm{H}(3 \mathrm{~A})$ & $4 e$ & 0.7680 & 0.1099 & 0.6157 & 0.047 \\
$\mathrm{H}(3 \mathrm{~B})$ & $4 e$ & 0.6626 & 0.1437 & 0.5932 & 0.047 \\
$\mathrm{H}(4 \mathrm{~A})$ & $4 e$ & 0.9133 & 0.0974 & 0.5350 & 0.047 \\
$\mathrm{H}(4 \mathrm{~B})$ & $4 e$ & 0.9423 & 0.0979 & 0.4373 & 0.047 \\
$\mathrm{H}(5 \mathrm{~N})$ & $4 e$ & 0.7826 & 0.3902 & 0.3788 & 0.045 \\
$\mathrm{H}(6 \mathrm{~A})$ & $4 e$ & 0.5754 & 0.1324 & 0.3442 & 0.047 \\
$\mathrm{H}(6 \mathrm{~B})$ & $4 e$ & 0.5572 & 0.1508 & 0.4440 & 0.047 \\
$\mathrm{H}(1 \mathrm{C})$ & $4 e$ & 0.8245 & -0.0938 & 0.2174 & 0.064 \\
$\mathrm{H}(1 \mathrm{D})$ & $4 e$ & 0.7106 & -0.0869 & 0.2405 & 0.064 \\
$\mathrm{H}(2 \mathrm{C})$ & $4 e$ & 0.7865 & -0.2650 & 0.3397 & 0.060 \\
$\mathrm{H}(2 \mathrm{D})$ & $4 e$ & 0.8696 & -0.1496 & 0.3739 & 0.060 \\
$\mathrm{H}(3 \mathrm{C})$ & $4 e$ & 0.8228 & -0.1562 & 0.5403 & 0.062 \\
$\mathrm{H}(3 \mathrm{D})$ & $4 e$ & 0.7333 & -0.2693 & 0.5237 & 0.062 \\
& & & & & \\
\hline
\end{tabular}


Table 2. Continued.

\begin{tabular}{llllll}
\hline Atom & Site & $x$ & $y$ & $z$ & $U_{\text {iso }}$ \\
\hline $\mathrm{H}(4 \mathrm{C})$ & $4 e$ & 0.6190 & -0.0890 & 0.5709 & 0.064 \\
$\mathrm{H}(4 \mathrm{D})$ & $4 e$ & 0.7031 & -0.1055 & 0.6514 & 0.064 \\
$\mathrm{H}(5 \mathrm{C})$ & $4 e$ & 0.9614 & 0.3360 & 0.4302 & 0.059 \\
$\mathrm{H}(5 \mathrm{D})$ & $4 e$ & 0.9822 & 0.3144 & 0.5391 & 0.059 \\
$\mathrm{H}(6 \mathrm{C})$ & $4 e$ & 0.8189 & 0.3757 & 0.5695 & 0.056 \\
& & & & & \\
\hline
\end{tabular}

Table 2. Continued.

\begin{tabular}{llllll}
\hline Atom & Site & $x$ & $y$ & $z$ & $U_{\text {iso }}$ \\
\hline $\mathrm{H}(6 \mathrm{D})$ & $4 e$ & 0.8581 & 0.5020 & 0.5033 & 0.056 \\
$\mathrm{H}(7 \mathrm{C})$ & $4 e$ & 0.6628 & 0.5288 & 0.4366 & 0.059 \\
$\mathrm{H}(7 \mathrm{D})$ & $4 e$ & 0.6446 & 0.4078 & 0.5145 & 0.059 \\
$\mathrm{H}(8 \mathrm{C})$ & $4 e$ & 0.5208 & 0.3770 & 0.3965 & 0.061 \\
$\mathrm{H}(8 \mathrm{D})$ & $4 e$ & 0.6012 & 0.3694 & 0.3192 & 0.061 \\
& & & & & \\
\hline
\end{tabular}

Table 3. Atomic coordinates and displacement parameters (in $\AA^{2}$ ).

\begin{tabular}{|c|c|c|c|c|c|c|c|c|c|c|}
\hline Atom & Site & $x$ & $y$ & $z$ & $U_{11}$ & $U_{22}$ & $U_{33}$ & $U_{12}$ & $U_{13}$ & $U_{23}$ \\
\hline $\mathrm{Ni}$ & $4 e$ & $0.74897(2)$ & $0.12645(3)$ & $0.43229(2)$ & $0.0255(2)$ & $0.0304(2)$ & $0.0315(2)$ & $0.0001(1)$ & $0.0016(1)$ & $-0.0009(1)$ \\
\hline $\mathrm{O}(1)$ & $4 e$ & $0.5704(2)$ & $0.6962(3)$ & $0.2894(1)$ & $0.051(1)$ & $0.088(2)$ & $0.062(1)$ & $0.011(1)$ & $0.021(1)$ & $-0.009(1)$ \\
\hline $\mathrm{O}(2)$ & $4 e$ & $0.4163(1)$ & $0.6658(2)$ & $0.3236(1)$ & $0.044(1)$ & $0.080(2)$ & $0.058(1)$ & $-0.016(1)$ & $-0.0040(9)$ & $-0.008(1)$ \\
\hline $\mathrm{O}(3)$ & $4 e$ & $0.5123(1)$ & $0.8113(3)$ & $0.4073(2)$ & $0.048(1)$ & $0.101(2)$ & $0.065(1)$ & $-0.015(1)$ & $0.007(1)$ & $-0.039(1)$ \\
\hline $\mathrm{O}(4)$ & $4 e$ & $0.9587(2)$ & $-0.0252(3)$ & $0.6600(2)$ & $0.075(2)$ & $0.160(3)$ & $0.046(1)$ & $0.028(2)$ & $0.021(1)$ & $-0.011(2)$ \\
\hline $\mathrm{O}(5)$ & $4 e$ & $0.8574(2)$ & $0.0708(3)$ & $0.7512(2)$ & $0.061(1)$ & $0.135(2)$ & $0.070(2)$ & $0.041(2)$ & $-0.002(1)$ & $-0.033(2)$ \\
\hline $\mathrm{O}(6)$ & $4 e$ & $0.9846(2)$ & $-0.0492(3)$ & $0.8064(2)$ & $0.102(2)$ & $0.139(2)$ & $0.066(2)$ & $0.031(2)$ & $-0.019(2)$ & $0.027(2)$ \\
\hline$N(1)$ & $4 e$ & $0.7889(2)$ & $0.0935(2)$ & $0.2886(1)$ & $0.040(1)$ & $0.050(1)$ & $0.037(1)$ & $0.001(1)$ & $0.0041(9)$ & $-0.001(1)$ \\
\hline$N(2)$ & $4 e$ & $0.7345(1)$ & $-0.1058(2)$ & $0.4255(1)$ & $0.035(1)$ & $0.034(1)$ & $0.044(1)$ & $-0.0015(9)$ & $-0.0020(9)$ & $-0.003(1)$ \\
\hline$N(3)$ & $4 e$ & $0.7148(2)$ & $0.0866(2)$ & $0.5773(1)$ & $0.035(1)$ & $0.048(1)$ & $0.036(1)$ & $0.004(1)$ & $0.0032(9)$ & $0.002(1)$ \\
\hline $\mathrm{N}(4)$ & $4 e$ & $0.9036(1)$ & $0.1434(2)$ & $0.4790(1)$ & $0.031(1)$ & $0.046(1)$ & $0.040(1)$ & $0.003(1)$ & $0.0019(9)$ & $-0.001(1)$ \\
\hline$N(5)$ & $4 e$ & $0.7624(1)$ & $0.3592(2)$ & $0.4359(1)$ & $0.040(1)$ & $0.035(1)$ & $0.037(1)$ & $-0.002(1)$ & $-0.0006(9)$ & $0.001(1)$ \\
\hline$N(6)$ & $4 e$ & $0.5961(1)$ & $0.1802(2)$ & $0.3974(2)$ & $0.032(1)$ & $0.042(1)$ & $0.043(1)$ & $-0.0011(9)$ & $-0.0005(9)$ & $-0.000(1)$ \\
\hline$N(7)$ & $4 e$ & $0.5003(2)$ & $0.7240(3)$ & $0.3394(2)$ & $0.039(1)$ & $0.053(1)$ & $0.039(1)$ & $0.005(1)$ & $-0.000(1)$ & $0.002(1)$ \\
\hline $\mathrm{N}(8)$ & $4 e$ & $0.9339(2)$ & $-0.0023(3)$ & $0.7394(2)$ & $0.040(1)$ & $0.064(2)$ & $0.038(1)$ & $0.003(1)$ & $0.005(1)$ & $-0.000(1)$ \\
\hline$C(1)$ & $4 e$ & $0.7782(2)$ & $-0.0671(3)$ & $0.2653(2)$ & $0.058(2)$ & $0.059(2)$ & $0.044(2)$ & $-0.002(2)$ & $0.009(1)$ & $-0.019(1)$ \\
\hline $\mathrm{C}(2)$ & $4 e$ & $0.7998(2)$ & $-0.1601(3)$ & $0.3528(2)$ & $0.045(2)$ & $0.038(2)$ & $0.066(2)$ & $0.007(1)$ & $0.004(1)$ & $-0.017(1)$ \\
\hline$C(3)$ & $4 e$ & $0.7523(2)$ & $-0.1646(3)$ & $0.5214(2)$ & $0.060(2)$ & $0.035(2)$ & $0.059(2)$ & $-0.005(1)$ & $-0.010(1)$ & $0.009(1)$ \\
\hline$C(4)$ & $4 e$ & $0.6897(2)$ & $-0.0735(3)$ & $0.5868(2)$ & $0.057(2)$ & $0.061(2)$ & $0.043(2)$ & $-0.015(2)$ & $0.004(1)$ & $0.010(1)$ \\
\hline$C(5)$ & $4 e$ & $0.9323(2)$ & $0.3025(3)$ & $0.4879(2)$ & $0.036(2)$ & $0.056(2)$ & $0.056(2)$ & $-0.012(1)$ & $-0.004(1)$ & $-0.005(1)$ \\
\hline$C(6)$ & $4 e$ & $0.8415(2)$ & $0.3965(3)$ & $0.5069(2)$ & $0.050(2)$ & $0.040(2)$ & $0.050(2)$ & $-0.010(1)$ & $-0.005(1)$ & $-0.007(1)$ \\
\hline$C(7)$ & $4 e$ & $0.6627(2)$ & $0.4222(3)$ & $0.4497(2)$ & $0.050(2)$ & $0.035(1)$ & $0.062(2)$ & $0.009(1)$ & $-0.002(1)$ & $-0.005(1)$ \\
\hline$C(8)$ & $4 e$ & $0.5881(2)$ & $0.3441(3)$ & $0.3839(2)$ & $0.044(2)$ & $0.046(2)$ & $0.063(2)$ & $0.015(1)$ & $-0.010(1)$ & $-0.002(1)$ \\
\hline
\end{tabular}

Acknowledgment. This work was supported by the Education Office of Hubei Province (2002B29002).

\section{References}

1. Karplus, P. A.; Pearson, M. A.: 70 years of crystalline urease: What have we learned? Acc. Chem. Res. 30 (1997) 330-337.

2. Lvov, Y.; Antipov, A. A.; Mamedov, A.; Mohwald, H.; Sukhorukov, G. B.: Urease encapsulation in nanoorganized microshells. Nano Lett. 1 (2001) 125-128.

3. Zhu, H.-L.; Zheng, L.-M.; Zhao, J.; Bu, W.-M.; Tang, W.-X.: Synthesis, Crystal Structure and Characterization of a Dinuclear Nickel(II) Complex with a Dinucleating Hexaazamacrocycle. Transition Met. Chem. 24 (1999) 131-134.

4. Zhu, H.-L.; Tong, Y.-X.; Chen, X.-M.; Ren, C.-X.: Synthesis, crystal structure and physical properties of two terephthalate-bridged copper(II) and nickel(II) complexes. Transition Met. Chem. 26 (2001) 528-531.

5. Sheldrick, G. M.: Siemens SHELXTL (Version 5.0): Siemens Industrial Automation, Inc, Analytical Instrumentation, Madinson, USA 1995. 